\title{
A new approach to sum frequency generation of single-frequency blue light in a coupled ring cavity
}

\author{
Jensen, Ole Bjarlin; Petersen, Paul Michael
}

Published in:

Proceedings of SPIE

Link to article, DOI:

$10.1117 / 12.2036328$

Publication date:

2014

Document Version

Publisher's PDF, also known as Version of record

Link back to DTU Orbit

Citation (APA):

Jensen, O. B., \& Petersen, P. M. (2014). A new approach to sum frequency generation of single-frequency blue light in a coupled ring cavity. In Proceedings of SPIE (Vol. 8964). SPIE. Proceedings of SPIE - The International Society for Optical Engineering https://doi.org/10.1117/12.2036328

\section{General rights}

Copyright and moral rights for the publications made accessible in the public portal are retained by the authors and/or other copyright owners and it is a condition of accessing publications that users recognise and abide by the legal requirements associated with these rights.

- Users may download and print one copy of any publication from the public portal for the purpose of private study or research.

- You may not further distribute the material or use it for any profit-making activity or commercial gain

- You may freely distribute the URL identifying the publication in the public portal

If you believe that this document breaches copyright please contact us providing details, and we will remove access to the work immediately and investigate your claim. 


\title{
A new approach to sum frequency generation of single- frequency blue light in a coupled ring cavity
}

\author{
Ole Bjarlin Jensen* and Paul Michael Petersen \\ DTU Fotonik, Department of Photonics Engineering, Technical University of Denmark, DK-4000 \\ Roskilde, Denmark
}

\begin{abstract}
We present a generic approach for the generation of tunable single-frequency light and demonstrate generation of more than $300 \mathrm{~mW}$ tunable light around $460 \mathrm{~nm}$. One tapered diode laser is operated in a coupled ring cavity containing the nonlinear crystal and another tapered diode laser is sent through the nonlinear crystal in a single pass. A high conversion efficiency of more than $25 \%$ of the single-pass laser is enabled by the high circulating power in the coupled cavity. The system is entirely self-stabilized with no need for electronic locking.
\end{abstract}

Keywords: tapered diode laser, coupled cavity, sum frequency generation, optical feedback

\section{INTRODUCTION}

Many applications within biophotonics and spectroscopy rely on visible laser sources with the possibility of selecting the desired wavelength. Such lasers can be challenging to develop with the exact requirements for the applications. Diode lasers in the visible spectral region are available in the red, green and blue. The output power from single-mode visible diode lasers is limited to about 100 $\mathrm{mW}$ and these lasers tend to operate in multiple longitudinal modes. Higher output power is possible using broad area diode lasers but at the expense of a decrease in spatial beam properties. Single-frequency output from visible diode lasers can be obtained from external cavity diode lasers or from diode laser with internal gratings. Up to $100 \mathrm{~mW}$ was recently demonstrated in the red spectral region at $635 \mathrm{~nm}$ using a DBR grating for frequency selection. Using internal gratings, however, inherently limits the tunability. Visible external cavity single-mode diode lasers typically emit lower output power.

Frequency doubling is a well-established method for generation of visible light by frequency conversion of near-infrared lasers. A lack of suitable gain media at various wavelengths limits the spectral coverage of second harmonic generation (SHG). Another very flexible source of tunable visible light is an optical parametric oscillator, but these have the disadvantage of being complicated to operate and require high pump power sources with excellent beam quality. Sum-frequency generation (SFG) has the possibility to generate light throughout most of the visible spectrum by proper choice of the fundamental laser wavelengths. A well-known application is for generation of light at the Sodium D2 resonance at $589 \mathrm{~nm}$. By mixing two diode pumped solid state lasers, tens and hundreds of $\mathrm{mW}$ can be generated in a single pass through a nonlinear crystal ${ }^{2}$, . SFG between a diode laser and a solid state laser was shown to generate light at $492 \mathrm{~nm}^{4}$ and recently generation of up to $4 \mathrm{~W}$ of green light at $531 \mathrm{~nm}$ was demonstrated by SFG between two beam combined DBR tapered diode lasers ${ }^{5}$. Although single-pass SFG is a simple and robust method for generation of visible light, the conversion efficiency is limited by the input power available from the interacting lasers. Higher conversion efficiency can be obtained by resonating one or both interacting beams in a high-finesse resonator. This can be achieved by placing the nonlinear crystal inside the cavity of a laser ${ }^{6}-{ }^{10}$ or in an external enhancement cavity ${ }^{11} .41 \%$ conversion efficiency of the single-pass laser has been demonstrated in this way efficiently generating yellow light ${ }^{7}$ and the possibility of generating almost any desired wavelength was demonstrated by Karamehmedovic et al. generating light at $488 \mathrm{~nm}$ by mixing a solid state laser and a tunable diode laser'. Higher conversion efficiency can be obtained by resonating both interacting waves inside a laser cavity ${ }^{12}$ or external cavity ${ }^{13}$, however at the expense of increased complexity and instability.

Recently coupled cavity operation of tapered amplifiers and SHG has been demonstrated to generate high output power and excellent beam quality in the blue and green spectral ranges ${ }^{14},{ }^{15}$. Skoczowsky et al. showed operation of a $976 \mathrm{~nm}$ tapered amplifier operated in a unidirectional ring cavity coupled to a compact ring cavity with the SHG material enclosed ${ }^{14}$. More than $300 \mathrm{~mW}$ of diffraction limited single-frequency output at $488 \mathrm{~nm}$ was obtained. In a similar approach, more than $500 \mathrm{~mW}$ of tunable green light was demonstrated ${ }^{15}$. The coupled cavity setups have the advantage of being entirely passivle locked by optical feedback to the tapered amplifiers.

*ojen@fotonik.dtu.dk; phone +45 46774553; fax +45 46774588; www.fotonik.dtu.dk

Nonlinear Frequency Generation and Conversion: Materials, Devices, and Applications XIII, edited by Konstantin L. Vodopyanov, Proc. of SPIE Vol. 8964, 896404 · c 2014 SPIE CCC code: $0277-786 X / 14 / \$ 18 \cdot$ doi: $10.1117 / 12.2036328$ 
Here, we present a generic approach for efficient generation of single-frequency visible light by SFG between two tapered lasers ${ }^{16}$. One external cavity tapered laser is single-passed through a nonlinear crystal contained in a coupled ring cavity containing the second tapered amplifier. In the coupled cavity, the power of one tapered laser is enhanced to increase the single-pass conversion efficiency to more than $25 \%$ resulting in generation of more than $300 \mathrm{~mW}$ of single-frequency light at $459 \mathrm{~nm}$. The resulting beam is diffraction limited due to nonlinear beam lean-up of the single-pass laser inside the nonlinear crystal. The laser system relies on optical feedback and no electronic locking is required. This approach can be expanded to cover the entire visible spectrum by proper choice of tapered amplifiers.

\section{EXPERIMENTAL SETUP}

The experiment was constructed from two different laser parts. One part was a coupled ring cavity tapered laser including the nonlinear frequency conversion crystal and the other part was an external cavity tapered laser which was single-passed through the nonlinear crystal to efficiently generate light at the sum frequency. A sketch of the experimental setup can be found in figure 1.

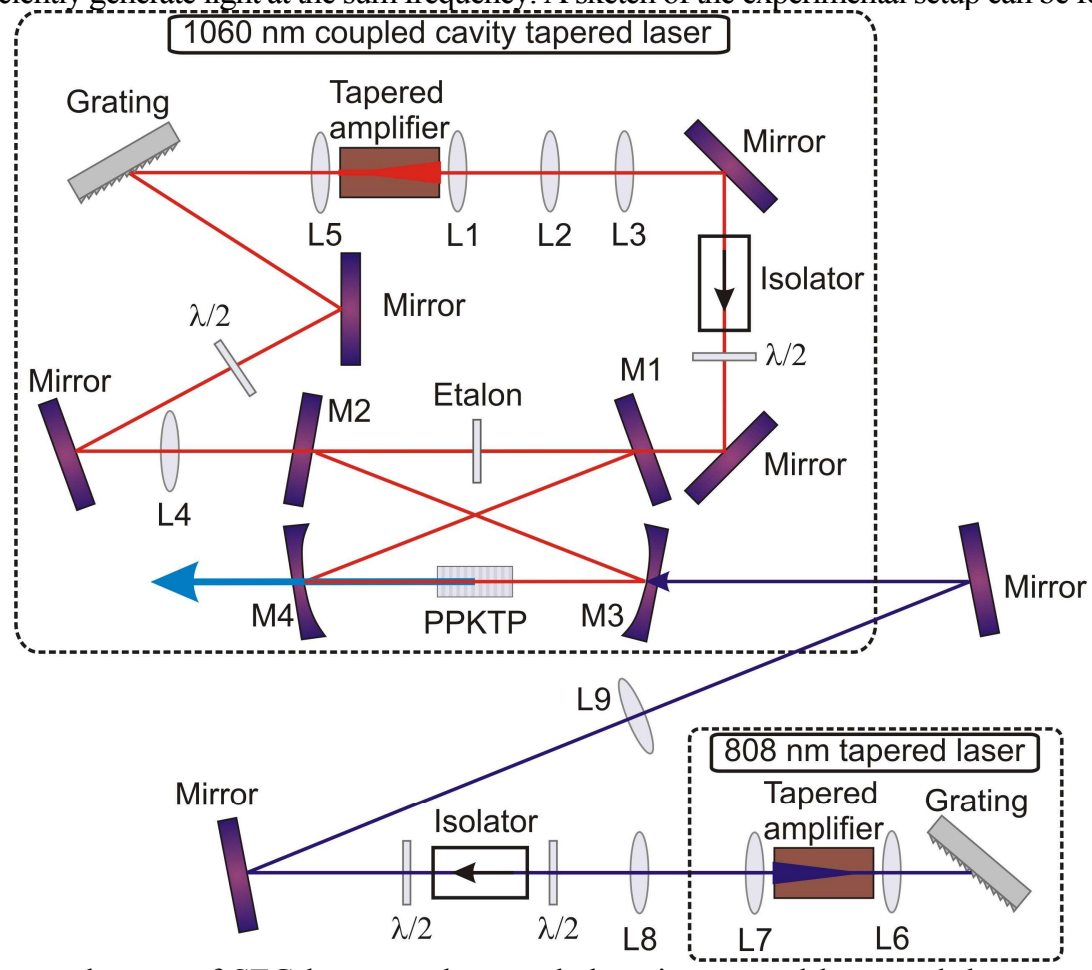

Figure 1. Experimental setup of SFG between the coupled cavity tapered laser and the external cavity tapered laser.

The main component of the coupled cavity tapered laser was the tapered amplifier. In the experiments, a $4 \mathrm{~mm}$ long tapered amplifier consisting of a $1 \mathrm{~mm}$ long ridge waveguide section and a $3 \mathrm{~mm}$ tapered section was used. The tapered section had an angle of $4^{\circ}$ giving an aperture width of $210 \mu \mathrm{m}$. The active region of the amplifier was constructed as a super large optical cavity ${ }^{17}$ resulting in a vertical far field angle of about $22^{\circ}$ (FWHM). The amplifier facets were passivated and coated to a rear facet reflectivity below $0.1 \%$ and a front facet reflectivity of about $2 \%$. The amplifier was first characterized in a Littrow external cavity setup ${ }^{18}$. At 3 A driving current, the laser was tunable in the $1049 \mathrm{~nm}-1093 \mathrm{~nm}$ wavelength range with a maximum output power of $1.79 \mathrm{~W}$ obtained at 1064 $\mathrm{nm}$. The power was higher than $1.6 \mathrm{~W}$ in the $1052 \mathrm{~nm}$ to $1080 \mathrm{~nm}$ wavelength range. The output beam along the slow axis had a strong central lobe enclosing about $80 \%$ of the output power and some low-intensity higher order modes. This resulted in a beam propagation factor of $\mathrm{M}^{2}=1.3$ and 2.8 in the fast and slow axis direction, respectively.

In the coupled ring cavity, the output from the tapered amplifier was collimated in the fast axis using an aspherical lens with a focal length of $3.1 \mathrm{~mm}$ (L1). A cylindrical lens with a focal length of $30 \mathrm{~mm}$ (L2) was used to correct for the astigmatism of the tapered amplifier and collimate the beam in the slow axis direction. A spherical lens (L3) with 250 $\mathrm{mm}$ focal length focused the beam into the enhancement cavity consisting of mirrors M1, M2, M3 and M4. A $30 \mathrm{~dB}$ optical isolator ensured unidirectional operation of the ring laser and a half-wave plate rotated the polarisation to 
horizontal as needed for efficient SFG in the nonlinear crystal. The four mirrors in the bow-tie enhancement cavity were coated as follows: M1 (plane) was coated for $95 \%$ reflectivity at $1064 \mathrm{~nm}, \mathrm{M} 2$ (plane) was coated for $98 \%$ reflectivity at $1064 \mathrm{~nm}, \mathrm{M} 3(\mathrm{R}=50 \mathrm{~mm})$ was coated for high reflectivity at $1064 \mathrm{~nm}$ and highly transmitting at $808 \mathrm{~nm}$ and M4 $(\mathrm{R}=$ $50 \mathrm{~mm}$ ) was highly reflecting at $1064 \mathrm{~nm}$ and highly transmitting at $532 \mathrm{~nm}$. M4 had a residual reflectivity at $459 \mathrm{~nm}$ of about $10 \%$. The cavity was constructed to support a beam waist diameter of $60 \times 70 \mu \mathrm{m}$ between the curved mirrors. A $10 \mathrm{~mm}$ long periodically poled KTP (PPKTP) crystal was positioned in this beam waist. The AR-coated PPKTP crystal was poled with a period of $5.6 \mu \mathrm{m}$ for SFG between $1064 \mathrm{~nm}$ and $808 \mathrm{~nm}$. A $125 \mu \mathrm{m}$ thick uncoated YAG etalon was included in the cavity to improve stability. The light exiting M2 was collimated using a $100 \mathrm{~mm}$ focal length lens (L4) and incident on a 1200 grooves $/ \mathrm{mm}$ grating for wavelength selection before coupled into the tapered amplifier through a $3.1 \mathrm{~mm}$ focal length lens (L5). A half-wave plate was included in order to optimize the power coupled to the tapered amplifier.

Operation of the tapered amplifier in this external cavity resulted in the same tuning range as in the Littrow setup. The output beam profile was purely TEM00 with a beam propagation factor of 1.03 , a result of the mode-cleaning properties of the cavity.

The $808 \mathrm{~nm}$ external cavity tapered laser was operated in the Littrow geometry with a 1200 grooves $/ \mathrm{mm}$ diffraction grating for wavelength selection ${ }^{18}$. The output was collimated in the fast axis using a $3.1 \mathrm{~mm}$ focal length aspherical lens (L7) and a $40 \mathrm{~mm}$ focal length cylindrical lens (L8). An optical isolator with $>30 \mathrm{~dB}$ isolation was inserted to prevent feedback to the tapered laser and two half-wave plates were used to control the power and polarization of the light incident on the PPKTP crystal. The light from the external cavity tapered laser was tunable in the range $792 \mathrm{~nm}-819$ $\mathrm{nm}(\mathrm{FWHM})$ with an output power of up to $1.28 \mathrm{~W}$ after the optical isolator. The beam propagation factor was measured to $\mathrm{M}^{2}=1.3 \times 1.9$ in the fast and slow axis respectively.

\section{EXPERIMENTAL RESULTS}

The circulating power in the enhancement cavity could be monitored by measuring the power leaking the cavity through mirrors M3 and M4. In this way it was found that the circulating power in the enhancement cavity can reach approximately $15 \mathrm{~W}$ when no light at $808 \mathrm{~nm}$ was incident on the PPKTP crystal.

The wavelength of the external cavity tapered laser was tuned to $807.5 \mathrm{~nm}$ and the coupled cavity tapered laser was tuned to $1063.5 \mathrm{~nm}$. These wavelengths allowed for phase matching of SFG in the PPKTP crystal at a temperature of $34^{\circ} \mathrm{C}$.

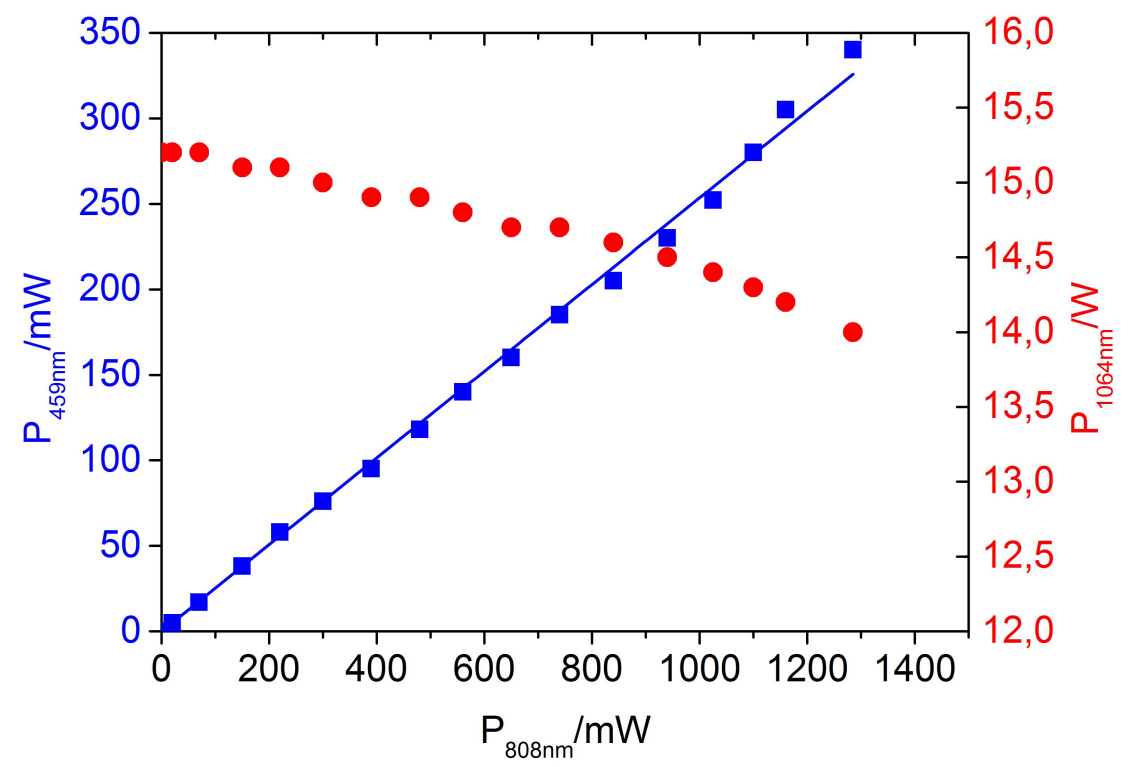

Figure 2. Measured output power at $459 \mathrm{~nm}$ (blue squares) and circulating power at $1064 \mathrm{~nm}$ (red dots) with varying input power at $807.5 \mathrm{~nm}$. 
At maximum input power of $1.28 \mathrm{~W}$ from the $807.5 \mathrm{~nm}$ tapered laser, an output power of $340 \mathrm{~mW}$ at $459 \mathrm{~nm}$ was measured with $14 \mathrm{~W}$ circulating power in the enhancement cavity. The power characteristics are shown in figure 2 . It can be seen that the generated output power at $459 \mathrm{~nm}$ increases approximately linearly with the input power at $807.5 \mathrm{~nm}$. It is further seen that the circulating power at $1063.5 \mathrm{~nm}$ decreased when the losses due to the SFG process increased. The losses induced by the SFG process increases linearly with the incident power as opposed to SHG where the losses increase quadratically. The extracted power of $340 \mathrm{~mW}$ was obtained without taking the losses of the nonoptimized components into account. The mirror M4 and the dichroic mirror were not optimized for $459 \mathrm{~nm}$ and when these losses are taken into account, more than $400 \mathrm{~mW}$ of generated light can be deduced. This corresponds to a single-pass conversion efficiency of more than $31 \%$ and a nonlinear conversion efficiency of $2.2 \% / \mathrm{W}$ in the PPKTP crystal.

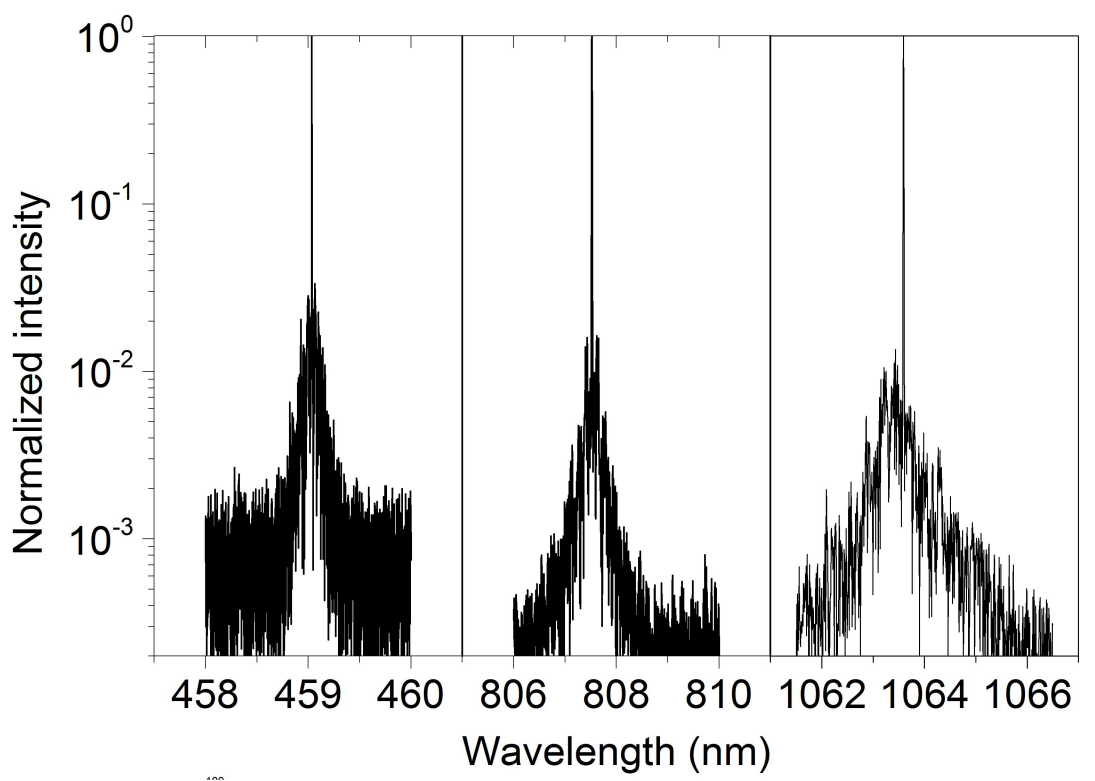

Figure 3. Measured spectra at $459 \mathrm{~nm}, 807.5 \mathrm{~nm}$ and $1063.5 \mathrm{~nm}$.

The spectrum of the light generated by SFG was determined by the spectral properties of the $807.5 \mathrm{~nm}$ laser and the $1063.5 \mathrm{~nm}$ laser. As both of these lasers are single-frequency, it is expected that the blue light is also single frequency. This was verified using an optical spectrum analyser and the linewidth measured at $459 \mathrm{~nm}$ was $2 \mathrm{pm}$ limited by the resolution of the optical spectrum analyser as shown in figure 3. To verify single-frequency operation of the two mixing lasers, scanning Fabry Perot interferometer (FPI) measurements were performed on the light at the two wavelengths. The FPI measurements are shown in figure 4, with a measured linewidth of below $12 \mathrm{MHz}$ at $807.5 \mathrm{~nm}$ and $10 \mathrm{MHz}$ at $1063.5 \mathrm{~nm}$, respectively. These linewidths were limited by the resolution of the FPIs. Mirrors for an FPI at $459 \mathrm{~nm}$ were not available but single-frequency operation at this wavelength can be deduced from the FPI measurements on the other wavelengths. 

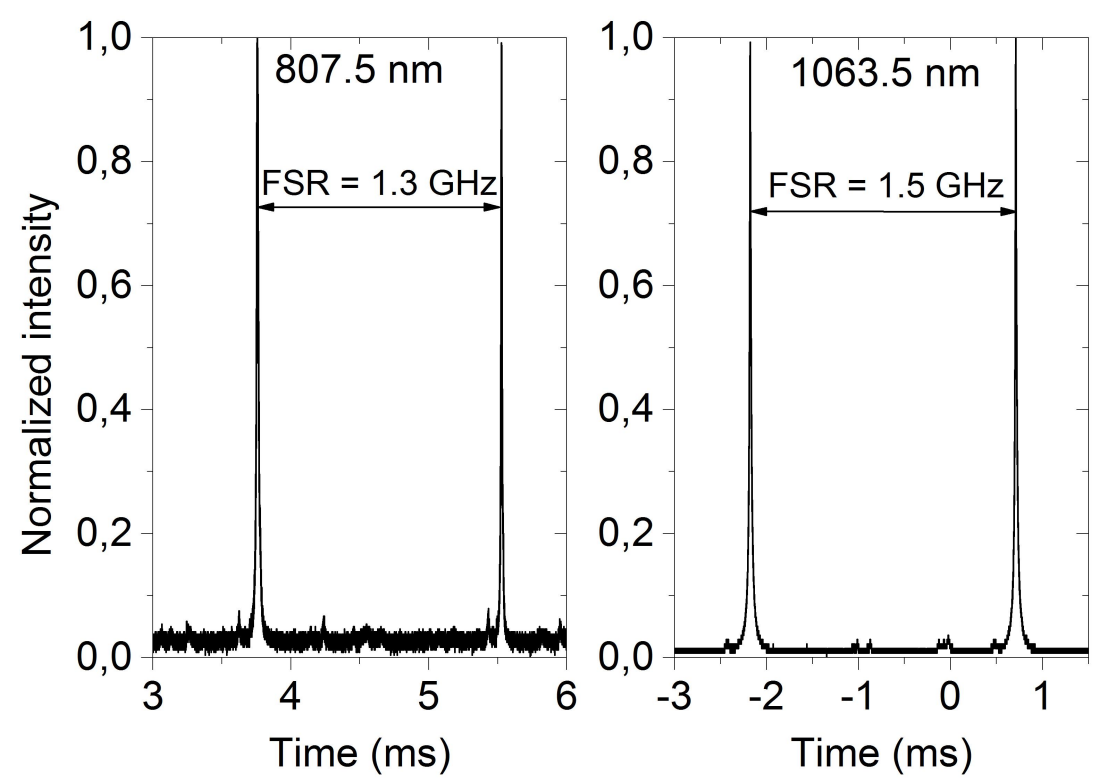

Figure 4. Scanning Fapry Perot measurements on light at $807.5 \mathrm{~nm}$ and $1063.5 \mathrm{~nm}$.

By selecting the tapered amplifiers to fit the desired generated wavelength, it is possible to generate light throughout the visible spectrum and also cover parts of the UV spectrum. Tuning of the generated light was demonstrated by tuning the $808 \mathrm{~nm}$ external cavity tapered laser. In this way, blue light in the $458.5 \mathrm{~nm}-460 \mathrm{~nm}$ range was generated. By fully exploiting the tunability of the two tapered amplifiers and using a different nonlinear crystal, a tuning range of $451 \mathrm{~nm}-$ $468 \mathrm{~nm}$ would be possible.

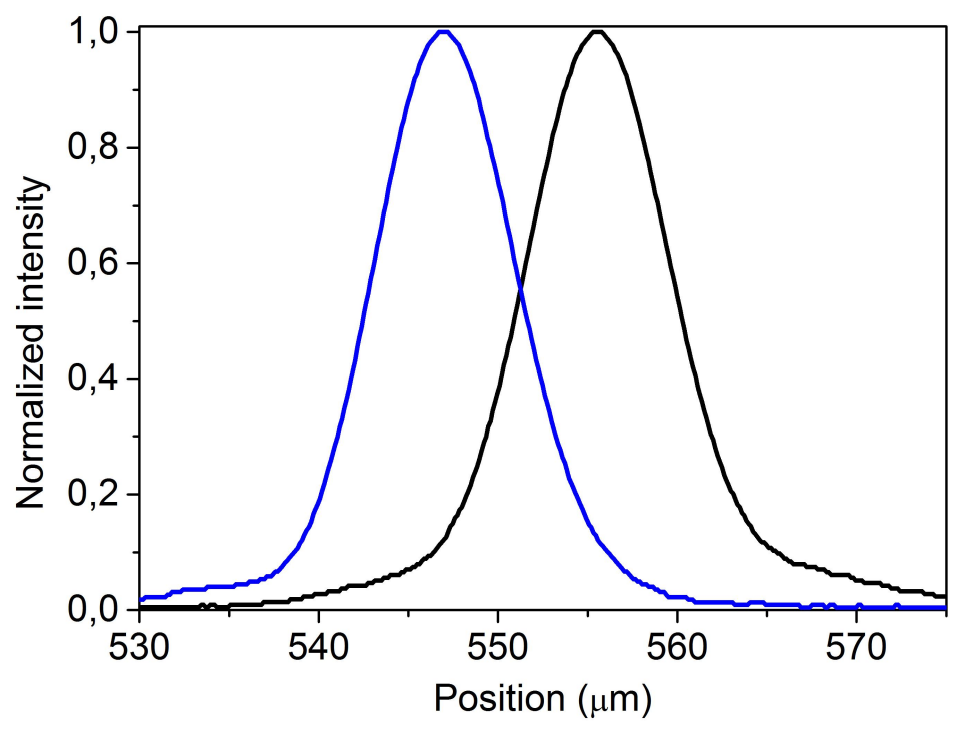

Figure 5. Beam profiles in the vertical and horizontal direction of the $459 \mathrm{~nm}$ light.

The spatial properties of the generated blue light are determined by a combination of the spatial properties of the two different mixing beams and by the overlap between the beams. Karamehmedović et al. showed that it is possible to use the SFG process between a Gaussian beam and a non-Gaussian beam to clean up the spatial properties by a proper selection of the overlap between the two beams $^{19}$. Here, we used the Gaussian beam in the enhancement cavity to clean up the near diffraction limited beam from the external 
cavity tapered laser. The result was a generated blue beam that was nearly Gaussian and close to diffraction limited with a beam propagation factor $\mathrm{M}^{2}<1.15$ in both directions as shown in figure 5 and figure 6.

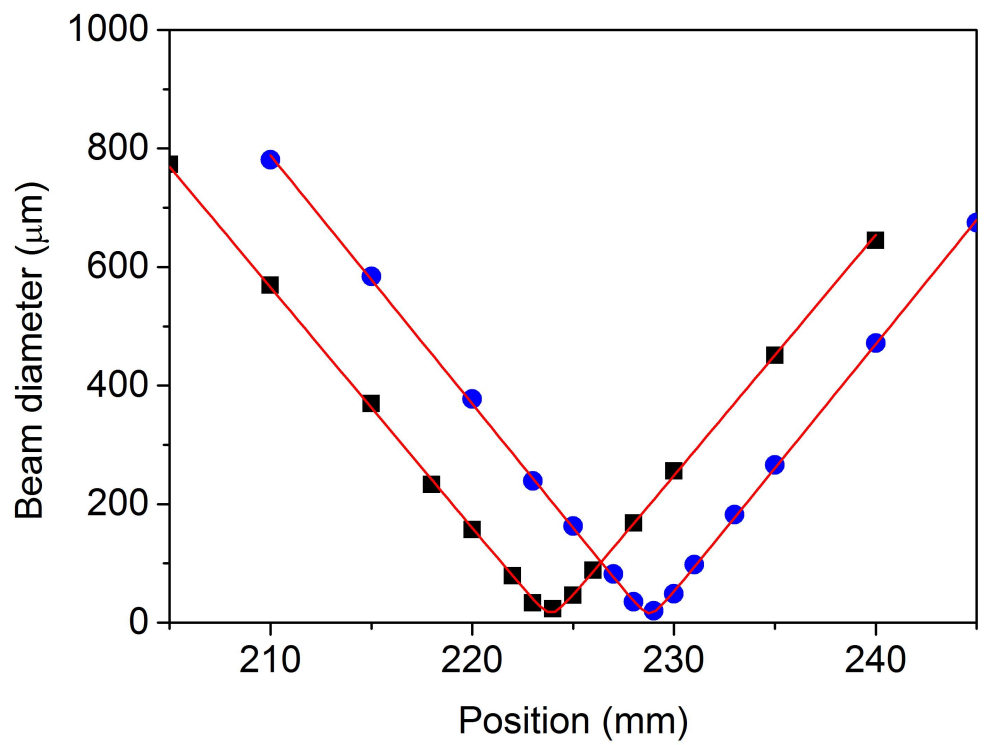

Figure 6. Measured beam widths for the $459 \mathrm{~nm}$ light.

\section{CONCLUSION}

A generic approach for generation of single-frequency tunable visible light has been demonstrated. An external cavity tapered laser was single-passed through a nonlinear crystal enclosed in a coupled ring cavity tapered laser. Efficient sum frequency generation was achieved in the nonlinear crystal as a result of the increased circulating power in the ring cavity. We applied this approach to two tapered amplifiers at $808 \mathrm{~nm}$ and $1064 \mathrm{~nm}$ to generate more than $300 \mathrm{~mW}$ of nearly diffraction limited blue light at $459 \mathrm{~nm}$. We demonstrated wavelength tuning of $1.5 \mathrm{~nm}$ and a significantly wider tuning range is possible by selecting a different nonlinear crystal. Other spectral regions can easily be reached by proper selection of tapered amplifiers.

\section{REFERENCES}

[1] Feise, D., John, W., Bugge, F., Blume, G., Hassoun, T., Fricke, J., Paschke, K., and Erbert, G., "96 mW longitudinal single mode red-emitting distributed Bragg reflector ridge waveguide laser with tenth order surface gratings.," Optics letters 37(9), 1532-4 (2012).

[2] Yue, J., She, C.-Y., Williams, B.P., Vance, J.D., Acott, P.E., and Kawahara, T.D., "Continuous-wave sodium D2 resonance radiation generated in single-pass sum-frequency generation with periodically poled lithium niobate.," Optics Letters 34(7), 1093-1095 (2009).

[3] Nishikawa, T., Ozawa, A., Nishida, Y., Asobe, M., Hong, F.-L., and Hänsch, T.W., "Efficient 494 mW sumfrequency generation of sodium resonance radiation at $589 \mathrm{~nm}$ by using a periodically poled $\mathrm{Zn}: \mathrm{LiNbO} 3$ ridge waveguide.," Optics Express 17(20), 17792-17800 (2009).

[4] Johansson, S., Spiekermann, S., Wang, S., Pasiskevicius, V., Laurell, F., and Ekvall, K., "Generation of turquoise light by sum frequency mixing of a diode-pumped solid-state laser and a laser diode in periodically poled KTP.," Optics Express 12(20), 4935-4940 (2004).

[5] Müller, A., Jensen, O.B., Hasler, K.-H., Sumpf, B., Erbert, G., Andersen, P.E., and Petersen, P.M., "Efficient concept for generation of diffraction-limited green light by sum-frequency generation of spectrally combined tapered diode lasers.," Optics Letters 37(18), 3753-3755 (2012). 
[6] Risk, W.P., Baumert, J.-C., Bjorklund, G.C., Schellenberg, F.M., and Lenth, W., "Generation of blue light by intracavity frequency mixing of the laser and pump radiation of a miniature neodymium:yttrium aluminum garnet laser," Applied Physics Letters 52(2), 85-87 (1988).

[7] Janousek, J., Johansson, S., Tidemand-Lichtenberg, P., Wang, S., Mortensen, J., Buchhave, P., and Laurell, F., "Efficient all solid-state continuous-wave yellow-orange light source.," Optics Express 13(4), 1188-1192 (2005).

[8] Sørensen, K.P., Tidemand-Lichtenberg, P., and Pedersen, C., "Efficient near diffraction limited blue light source by sum-frequency mixing of a BAL and a solid-state laser," Laser Physics Letters 8(3), 209-213 (2011).

[9] Karamehmedovic, E., Pedersen, C., Andersen, M.T., and Tidemand-Lichtenberg, P., "Efficient visible light generation by mixing of a solid-state laser and a tapered diode laser.," Optics Express 15(19), 12240-12245 (2007).

[10] Risk, W.P., and Lenth, W., "Diode laser pumped blue-light source based on intracavity sum frequency generation," Applied Physics Letters 54(9), 789-791 (1989).

[11] Vasilyev, S., Nevsky, A., Ernsting, I., Hansen, M., Shen, J., and Schiller, S., "Compact all-solid-state continuous-wave single-frequency UV source with frequency stabilization for laser cooling of $\mathrm{Be}+$ ions," Applied Physics B 103(1), 27-33 (2011).

[12] Kean, P.N., Standley, R.W., and Dixon, G.J., "Generation of $20 \mathrm{~mW}$ of blue laser radiation from a diodepumped sum-frequency laser," Applied Physics Letters 63(3), 302-304 (1993).

[13] Mimoun, E., De Sarlo, L., Zondy, J.-J., Dalibard, J., and Gerbier, F., "Sum-frequency generation of $589 \mathrm{~nm}$ light with near-unit efficiency.," Optics Express 16(23), 18684-18691 (2008).

[14] Skoczowsky, D., Jechow, A., Menzel, R., Paschke, K., and Erbert, G., "Efficient second-harmonic generation using a semiconductor tapered amplifier in a coupled ring-resonator geometry.," Optics Letters 35(2), 232-234 (2010).

[15] Jensen, O.B., and Petersen, P.M., "Generation of single-frequency tunable green light in a coupled ring tapered diode laser cavity," Optics Express 21(5), 6076-6081 (2013).

[16] Jensen, O.B., and Petersen, P.M., "Single-frequency blue light generation by single-pass sum-frequency generation in a coupled ring cavity tapered laser," Applied Physics Letters 103, 141107 (2013).

[17] Knauer, A., Erbert, G., Staske, R., Sumpf, B., Wenzel, H., and Weyers, M., "High-power 808 nm lasers with a super-large optical cavity," Semiconductor Science and Technology 20(6), 621-624 (2005).

[18] Chi, M., Jensen, O.B., Holm, J., Pedersen, C., Andersen, P.E., Erbert, G., Sumpf, B., and Petersen, P.M., "Tunable high-power narrow-linewidth semiconductor laser based on an external-cavity tapered amplifier," Optics Express 13(26), 10589-10596 (2005).

[19] Karamehmedović, E., Pedersen, C., Jensen, O.B., and Tidemand-Lichtenberg, P., "Nonlinear beam clean-up using resonantly enhanced sum-frequency mixing," Applied Physics B 96(2-3), 409-413 (2009). 